\title{
Torsional Fretting Wear Behavior of Duplex DLC Coatings Deposited on Ion Nitriding Treated LZ50 Steel
}

\author{
Zhen-bing Cai · He-geng Gu • Wen Yue • \\ Min-hao Zhu
}

Received: 20 November 2014/Revised: 6 February 2015/ Accepted: 11 February 2015/Published online: 27 February 2015

(C) Springer International Publishing AG 2015

\begin{abstract}
Duplex ion nitriding/DLC coatings were prepared on LZ50 steel. Characterizations were detected by scanning electron microscopy, atomic force microscope, Raman spectroscopy, dynamic ultra-micro hardness tester, and profilometer. The torsional fretting wear behaviors of DLC coatings and substrate LZ50 steel against GCr15 steel ball in dry conditions were studied under angular displacement amplitudes within a range of $0.1^{\circ}-5^{\circ}$, constant normal load of $50 \mathrm{~N}$, and rotational speed of $0.8^{\circ} \mathrm{s}^{-1}$. DLC coatings presented dense structure and high hardness. Compared with the substrate, DLC coatings removed the mix fretting regime and shifted the slip regime to low angular displacement amplitudes. The friction torques of DLC coatings were lower than that of the substrates. In the partial slip regime, the damage of DLC coatings was low. In the slip regime, the damage mechanism combined with delamination, abrasive wear, and graphitization. The DLC coatings exhibited good capability for anti-torsional fretting damage.
\end{abstract}

Keywords DLC coatings - Friction and wear · Fretting wear . Torsional fretting

Z. Cai $(\bowtie) \cdot H . \mathrm{Gu} \cdot$ M. Zhu

Key Lab of Advanced Technologies of Materials (Ministry of Education), Tribology Research Institute, Southwest Jiaotong University, Chengdu 610031, China

e-mail: czb-jiaoda@126.com

H. Gu

Ningbo Branch, Ordnance Science Institute of China, Ningbo 315103, China

W. Yue

School of Engineering and Technology, China University of Geosciences, Beijing 100083, China

\section{Introduction}

Fretting is caused by the relative oscillatory movement of small amplitudes that may occur between contacting surfaces subjected to vibration; this phenomenon may lead to service failure because of rapid crack formation and surface wear [1-4]. According to relative motion directions for a ball-on-flat contact, four fundamental fretting modes exist, i.e., tangential, radial, torsional, and rotational modes [5-9]. Torsional fretting is the relative motion induced by the reciprocating torsion in an oscillatory vibratory environment [7]. Torsional fretting is common in aeronautics, rail transport, artificial implant engineering, etc., such as ball sockets in automobiles, wheel-axle fits in trains, and human joints [10-13]. Therefore, measures to reduce or eliminate torsional fretting damage are necessary.

The use of surface engineering techniques as effective methods to improve fretting resistance has attracted the attention of many researchers [14-16]. Among surface engineering techniques, significant attention has shifted to applying diamond-like carbon (DLC) coating because of its tribological properties, such as low friction, high hardness, high wear resistance, high elastic modulus, and chemical stability [17-20]. However, DLC films deposited on "soft" substrates (e.g., axle steel) cannot obtain enough support for hard DLC films; these films spill from the substrate, adversely affect tribological performance and durability, and largely limit the application of DLC films [17]. Forming a hard middle layer on soft substrate materials, i.e., nitriding or carburizing before depositing DLC films will have beneficial tribological behavior. The ion nitriding process is a good pretreatment method for hardening the substrate. This process has been widely used in steel to improve the surface and friction properties and the load-bearing capacity of dynamically loaded components [21-25]. 
Few studies have focused on the fretting behavior of DLC, not to mention torsional fretting wear. In this research, ion nitriding plus depositing DLC films duplex treatments was completed for LZ50 steel. Torsional fretting wear behavior of LZ50 steel substrate and nitriding/DLC samples was studied, and interesting findings were foun$\mathrm{d}$ and discussed.

\section{Materials and Experimental Detail}

\subsection{Materials}

In this paper, railway axle steel-LZ50 steel [(wt\%): $0.55 \mathrm{C}$, $0.3 \mathrm{Si}, 0.72 \mathrm{Mn}, 0.3 \mathrm{Ni}, 0.3 \mathrm{Cr}$ ] and Fe samples with dimensions of $10 \mathrm{~mm} \times 10 \mathrm{~mm} \times 30 \mathrm{~mm}$ were used as substrates for coating preparation. The samples were machined after being sanded and polished. The average surface roughness was $R_{\mathrm{a}}=0.05 \mu \mathrm{m}$. The GCr15 ball with diameter of $40 \mathrm{~mm}$ and surface roughness of $0.02 \mu \mathrm{m}$ was used as the against ball specimen in the fretting wear test.

\subsection{Ion Nitriding and Ion Beam Auxiliary Deposition of DLC}

Ion nitriding was conducted on LZ50 using the LDM2-25 plasma nitriding furnace. Treatment parameters were ammonia $\left(\mathrm{NH}_{3}\right)$ as gases source, process pressure of $670 \mathrm{~Pa}$, process temperature of $520^{\circ} \mathrm{C}$, and duration of $4 \mathrm{~h}$. Thickness of the nitrided layers on the test sample was nearly $20 \mu \mathrm{m}[23,26]$.

DLC films were deposited on the nitrided LZ50 steel by ion beam-assisted deposition (IBAD) using ISB700 multifunctional ion beam sputtering coater. Initially, the nitrided LZ50 steel samples were sputter cleaned with Ar ion beam to remove adhering impurities on the surface, and the $\mathrm{Cr}$ ion was deposited on the samples for $2 \mathrm{~h}$ to obtain the interlayer. Finally, a graphite target (99.99 \%) was sputtered by $\mathrm{Ar}^{+}$ions at a beam voltage of $2.7 \mathrm{kV}$ with a total beam current of $100 \mathrm{~mA}$ and the deposited DLC coatings were simultaneously bombarded with $\mathrm{Ar}^{+}$ions at a beam voltage of $0.2 \mathrm{kV}$ with a total beam current of $20 \mathrm{~mA}$. The pressure of the chamber was $1.1 \times 10^{-2} \mathrm{~Pa}$ during the deposition, and the process lasted $2 \mathrm{~h}$.

\subsection{Torsional Fretting Wear Test and Analysis Method}

The torsional fretting wear test of LZ50 samples and nitriding/DLC samples was conducted on a fretting wear test rig [10]. Test parameters are constant rotary angular velocity $\omega=0.8^{\circ} \mathrm{s}^{-1}$, torsional angular displacement amplitude varied from $\theta=0.1^{\circ}$ to $5^{\circ}$, test cycles varied from 1 to $10^{4}$, and a constant normal load $F_{n}=50 \mathrm{~N}$. All tests were performed at $25{ }^{\circ} \mathrm{C}$ in ambient air $(\mathrm{RH}=60 \%)$, and every test was replaced three times.

Before the torsional fretting war tests, the cross section of the DLC coatings was observed under a scanning electron microscope (SEM, QUANTA2000) with energy-dispersive X-ray spectroscopy (EDX, EDAX-7760/68 M). The surface morphology of the DLC coatings was observed by an atomic force microscope (SPI 3800). The structure was characterized by Raman spectroscopy (Renishaw), and the dynamic ultra-micro hardness tester (DUH-211S) measured the hardness and elastic modulus.

After the torsional fretting wear tests, wear scars were examined by SEM. The profile of the wear scars was measured by a 3D surface profilometer (Aep, NanoMapD). The structures of the wear zones were detected by Raman spectroscopy.

\section{Results and Discussion}

\subsection{Characteristics of the Coatings}

Figure 1a shows that the surface of DLC coating was dense and uniform. The measurement results for the hardness and elastic modulus of the DLC coating are presented in Fig. 1b. The indentation hardness and indentation elastic modulus of the DLC coating were 26.3 and $315 \mathrm{GPa}$, respectively. The DLC films normally have hardness of $90 \mathrm{GPa}$ and modulus of $500 \mathrm{GPa}$ [27]. The results of this study are smaller than the average numbers because of the thinness $(300 \mathrm{~nm})$ and because the substrate contributed to the reduced measurement.

Element distributions of the cross section of the DLC coating under SEM + EDX observation are shown in Fig. 1c. The thickness of the DLC coating was approximately $300 \mathrm{~nm}$, as estimated by the cross-sectional image [b]. Figure 1d illustrates a typical Raman spectrum in the range of $800-2000 \mathrm{~cm}^{-1}$ of the DLC coating. The Gaussian fitting result of the coating indicates that the spectrum is composed of two broad bands centered at approximately 1574.9 (G band) and $1383.5 \mathrm{~cm}^{-1}$ (D band). The assignment of the $\mathrm{D}$ and $\mathrm{G}$ peaks is straightforward in the "molecular" picture of carbon materials. The G peak is due to the bond stretching of all pairs of $\mathrm{sp}^{2}$ atoms in both rings and chains. The $\mathrm{D}$ peak is due to the breathing modes of $\mathrm{sp}^{2}$ atoms in rings [26]. The ratio of $I_{\mathrm{D}} / I_{\mathrm{G}}$ of the DLC coating was approximately 4.31. This finding confirmed that the deposited coating was amorphous DLC

\subsection{Kinetic Behavior of Torsional Fretting}

Under test control conditions for angular displacement amplitude, the torsional fretting wear tests of the DLC 
Fig. 1 Characteristics of the DLC coating. a AFM surface morphology. b Microhardness and elastic modulus. c Crosssectional image with element distributions. d Raman spectrum

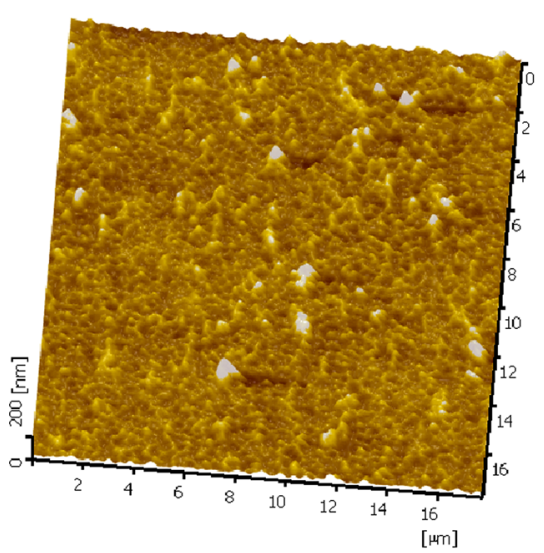

(a)

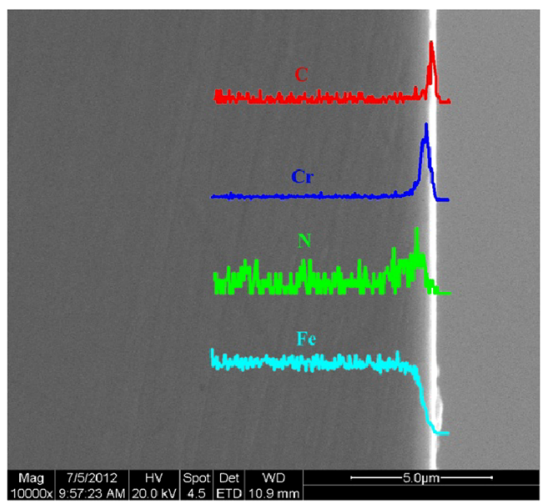

(c)

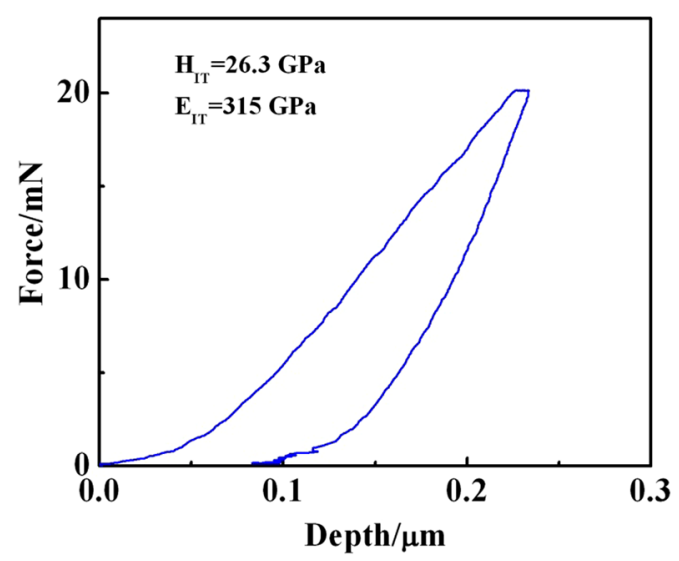

(b)

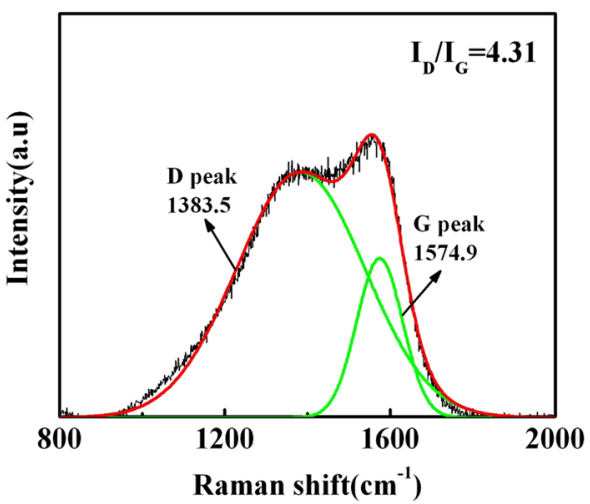

(d) coatings and LZ50 steel against GCr15 steel balls were conducted. The curves of the friction torque versus angular displacement amplitudes ( $T-\theta$ curves) were analyzed. Figure 2 shows the $T-\theta$ curves of the DLC coating and LZ50 steel as functions of the number of cycles under varied angular displacement amplitudes $\left(\theta=0.1^{\circ}, 0.5^{\circ}, 1^{\circ}\right.$, $2.5^{\circ}, 5^{\circ}$ ). Similar to tangential fretting, three basic types of $T-\theta$ curves, namely, linear, elliptic, and parallelogram loops, can be seen in Fig. 2. When the angular displacement amplitude was low $\left(\theta=0.1^{\circ}\right)$, the $T-\theta$ curves of the DLC coating and LZ50 steel all formed the shape of a line during the whole cycles, thus indicating that the contact interface was coordinated by elastic deformation and that fretting ran in the partial slip regime (PSR). When $\theta$ increased to $0.5^{\circ}$ because of the adsorption film on the surface the $T-\theta$ curve of LZ50 at the first cycle formed a parallelogram, and the shape gradually turned elliptic from the 10th to 100th cycle. This finding indicated that contact interface was coordinated with the elastic and plastic during that time. Thereafter, the shape changed to a parallelogram until the end of the test and the third body was formed. Fretting was transferred from partial slip status to gross slip status without considering the first 10 cycles (running-in stage), thus revealing that fretting ran in the mixed fretting regime. However, the $T-\theta$ curves of the DLC presented a parallelogram from the initial stage to the end of test. According to the variation of the $T-\theta$ curves and the evolution of the damage morphologies [10], i.e., in the mixed regime, the wear zone increased. Furthermore, the sticking zone diminished gradually as a function of the number of cycles. In the slip regime (SR), wear morphology completely covered the worn zone during entire cycles.

Figure 3 shows that when $\theta=0.5^{\circ}$, wear optical morphology (OM) of the DLC coatings covered the whole worn zone at the 100th cycle. Fretting ran in SR. When $\theta \geq 1^{\circ}$, the $T-\theta$ curves of LZ50 steel and DLC coatings were presented in the shape of a parallelogram and fretting ran in gross slip status during the whole cycles. However, the fretting of LZ50 steel ran in SR after $\theta \geq 5^{\circ}$. Table 1 lists the torsional fretting regime distributions. The MFR disappeared and the SR of the coating shifted to the direction of the lower angular displacement amplitude for the DLC coatings compared with those of LZ50 steel. This result is attributed to the mechanochemical interactions between the contact interfaces [6]. 


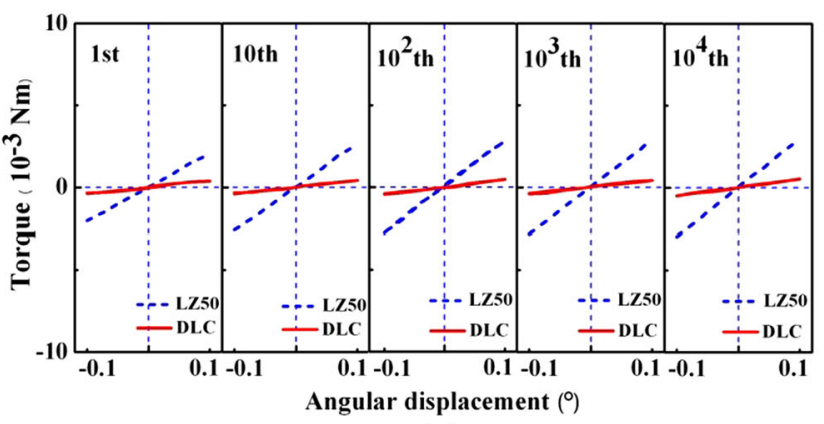

(a)

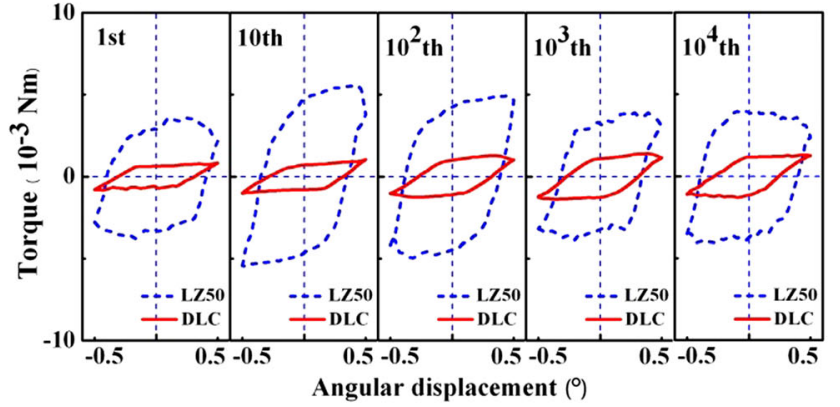

(b)

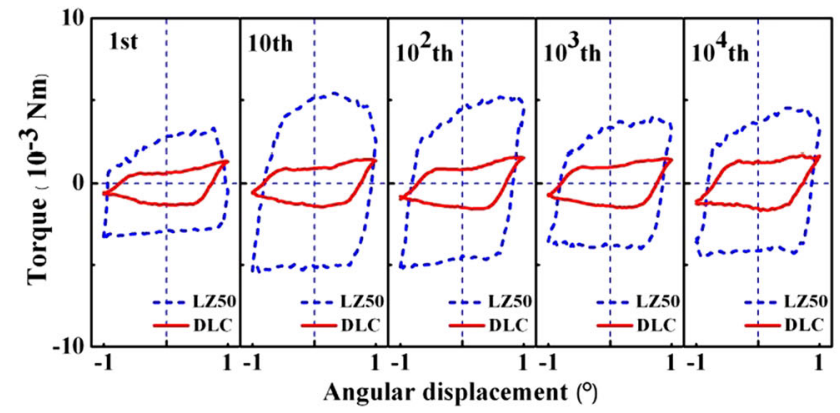

(c)

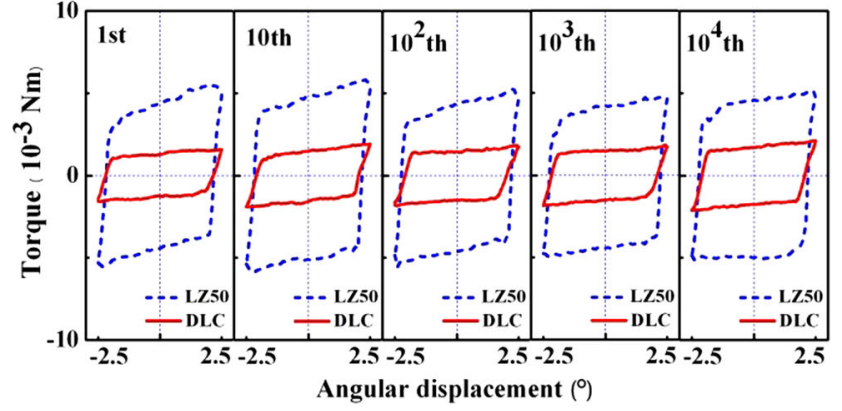

(d)

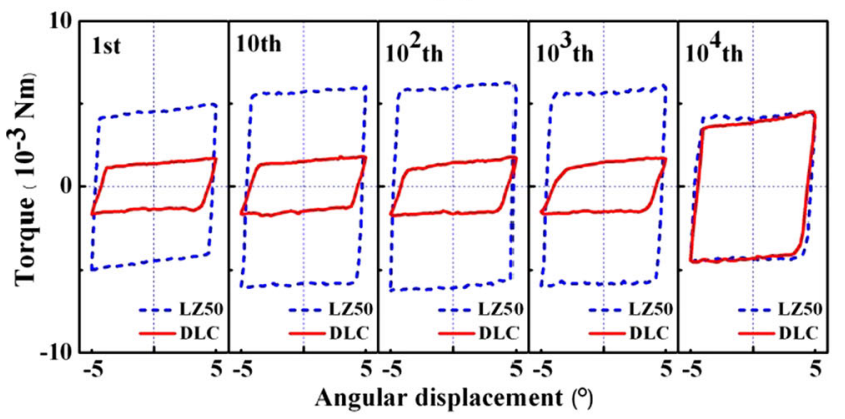

(e)

Fig. $2 T-\theta$ curves of the DLC coating and nitride layer under different angular displacement amplitudes. a $\theta=0.1^{\circ}, \mathbf{b} \theta=0.5^{\circ}, \mathbf{c} \theta=1^{\circ}$, d $\theta=2.5^{\circ}$, e $\theta=5^{\circ}$

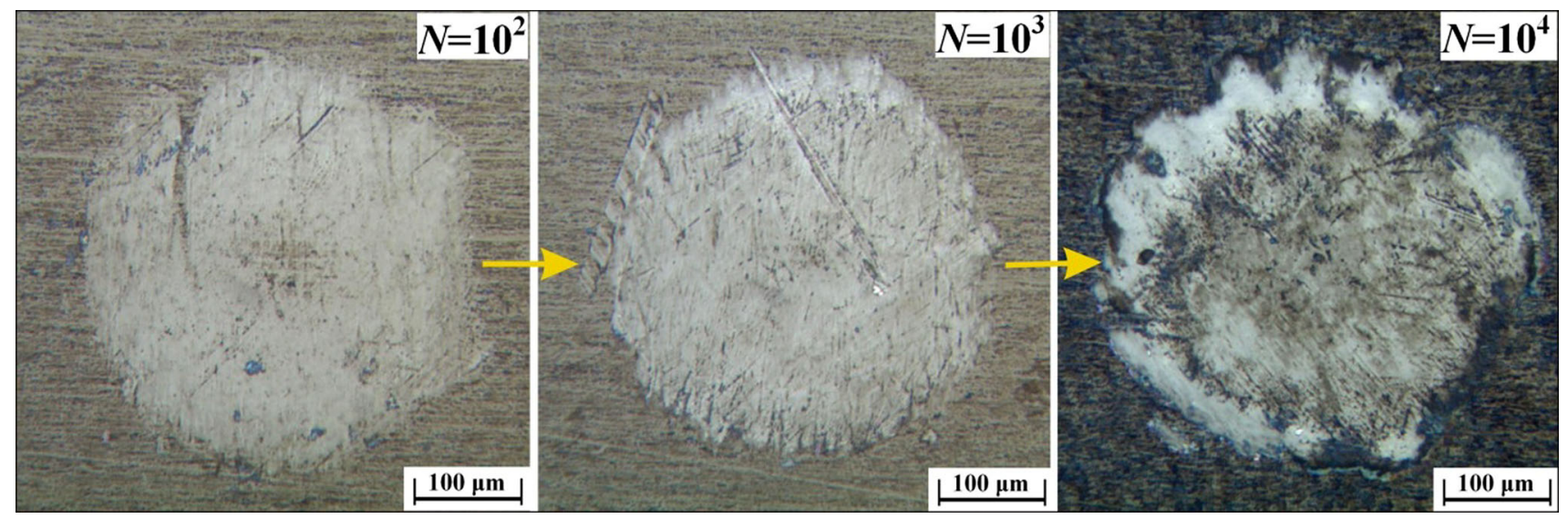

Fig. 3 Morphologies of the DLC wear scars in varied cycles: $\theta=0.5^{\circ}$ 
Table 1 Torsional fretting running regime of LZ50 steel and DLC coatings

\begin{tabular}{llllll}
\hline Angular displacement amplitude $\theta\left(^{\circ}\right)$ & 0.1 & 0.5 & 1 & 2.5 & 5 \\
\hline LZ50 & PSR & SR & SR & SR & SR \\
DLC & PSR & MFR & MFR & MFR & SR \\
\hline
\end{tabular}

$P S R$ partial slip regime, $M F R$ mixed regime, $S R$ gross slip regime

\subsection{Friction Torque}

Figure 4 shows the evolution curves of the friction torque for DLC coatings and LZ50 steel as functions of the number of cycles under angular displacement amplitude. An obvious difference is evident in the behavior between the DLC coatings and its substrate. In PSR $\left(\theta=0.1^{\circ}\right)$, the friction torque for the DLC coating presented a continued stable status at a low level, whereas the friction torque of its substrate was in the slow ascendant stage. The friction torque of the DLC coatings was evidently lower than that of the substrate (Fig. 4a). In MFR, for LZ50 steel
Fig. 4 Friction torque of the LZ50 steel and the DLC coatings under various torsional angular displacement amplitudes
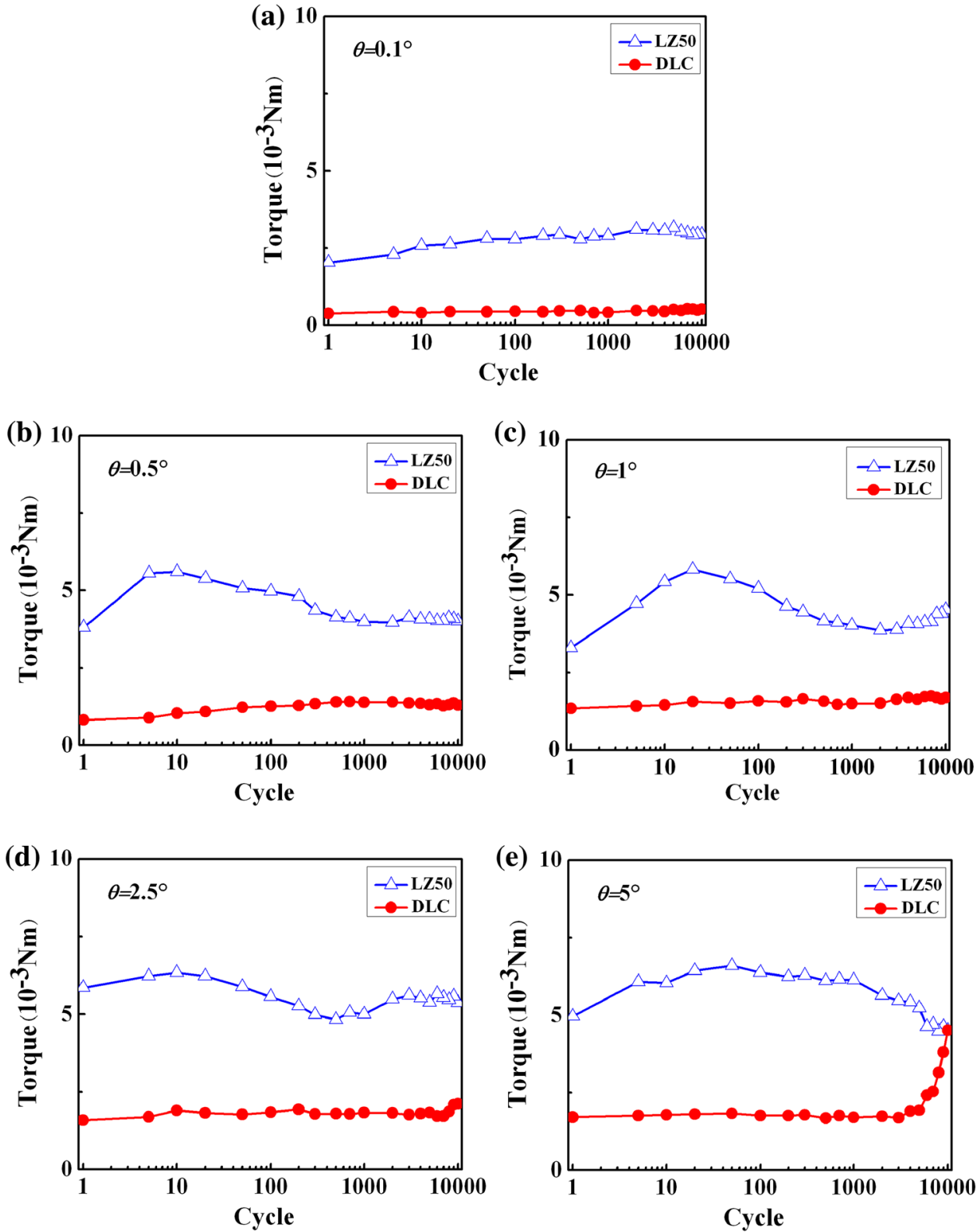
$\left(\theta=0.5^{\circ}, 1^{\circ}\right.$, and $\left.2.5^{\circ}\right)$, the friction torque presented three stages. In Stage I, the friction torque rapidly climbed to the maximum owing to the failure of the adsorbed and polluted surface films before approximately 10 cycles. In Stage II, strong plastic flow and surface hardworking occurred in the contact interface, thus increasing the friability of the surface material and delamination. Debris was gradually generated in the contact interface, wherein the two-body contact of GCr15-LZ50 steel turned to the three-body contact of GCr15-debris-LZ50 steel. Here, friction torque descended from peak value. In Stage III, the production and transfluence of debris came to a balanced state. The friction torque achieved a steady state (Fig. 4b-d). However, the mix fretting regime disappeared in the DLC coatings.

In $\mathrm{SR}$, the trend of friction torque for LZ50 steel $\left(\theta=5^{\circ}\right)$ was consistent with that in MFR but is delayed in achieving a steady state, which occurred only after about 6000 cycles (Fig. 4e). For DLC coatings, when $\theta=0.5^{\circ}$ and $1^{\circ}$, the torque kept a steady value, climbed to higher values between 1000 and 2000 cycles, and sustained the process to the end (Fig. 4b, c). This behavior could be due to the looseness between DLC coatings and nitride layer in relatively high peaks. When $\theta=2.5^{\circ}$ and $5^{\circ}$, similar to lower torque in SR, torque values were low and stable before 1000 cycles. The values then climbed rapidly and closed to that of its substrate at last. Such a behavior is the result of DLC coatings being detaching from the nitride layer in contact interface, even if DLC coatings were complete failures at $\theta=5^{\circ}$ (Fig. 4d, e), which would be discussed in detail in the following section.

Figure 5 shows the friction torque of the DLC coatings under various angular displacement amplitudes. Friction torque was low because of elastic deformation instead of relative motion on the contact interface in PSR. However, the values of friction torque at different angular displacement amplitudes were the same before failure because sliding easily occurred on the surface of DLC coatings.

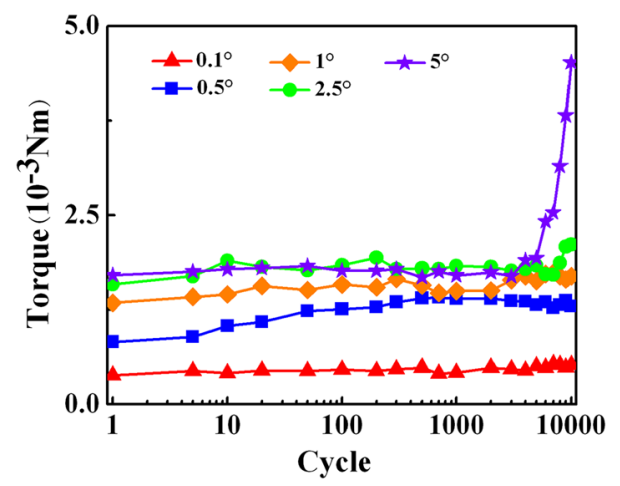

Fig. 5 Friction torque of the DLC coatings under various torsional angular displacement amplitudes
Furthermore, friction torque of DLC coatings was lower than that of LZ50 steel when $\theta=5^{\circ}$.

\subsection{Friction Dissipation Energy}

Friction dissipation energy, as one of the most important parameters, was used to evaluate damage. Siegfried Fouvry et al. [28, 29] found that fretting wear volume could be correlated with friction dissipation energy in a linear relationship. Therefore, to a certain extent, wear volume could be judged by their friction dissipation energy in fretting.

For torsional fretting, friction dissipation energy could be defined as the area of the loop encircled by close $T-\theta$ curve, mathematically [30],

$E=\sum_{n} E_{i}=\sum_{n} \int_{-\theta}^{\theta} T * \theta \mathrm{d} \theta$

where $E$ is friction dissipation energy, $T$ is friction torque, and $\theta$ is angular displacement amplitude in radian. Figure 6 reveals friction energy curves of DLC coatings and LZ50 steel as function of the number of cycles under different various angular displacement amplitudes. Friction energy curves of LZ50 steel and DLC coatings increased with increasing angular displacement amplitude. This trend was consistent with their $T-\theta$ curves. DLC coatings were lower than that of LZ50 steel, which indicated that wear volume of DLC coatings was less than that of LZ50 steel.

\subsection{Wear Analyses}

Like the other three fretting modes, the damage of the contact interface was greatly influenced by the angular displacement amplitudes [9]. In PSR $\left(\theta=0.1^{\circ}\right)$, slight damage occurred at the edge contact interfaces of the LZ50 steel and DLC coatings, and no damage appeared in the center of contact interface (Fig. 7). This result was due to the fretting being controlled by elastic deformation and was

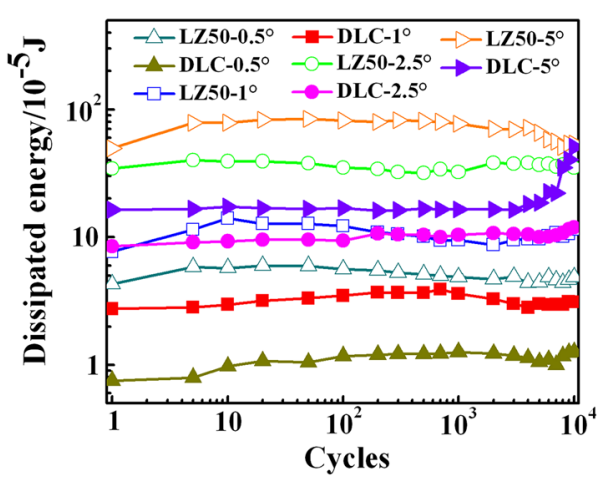

Fig. 6 Friction dissipated energy curves of DLC coatings and LZ50 steel as function of the cycles 
Fig. 7 SEM morphologies of the wear scars of LZ50 steel $\mathbf{a}$ and DLC coatings $\mathbf{b}$ in PSR $\left(\theta=0.1^{\circ}\right)$

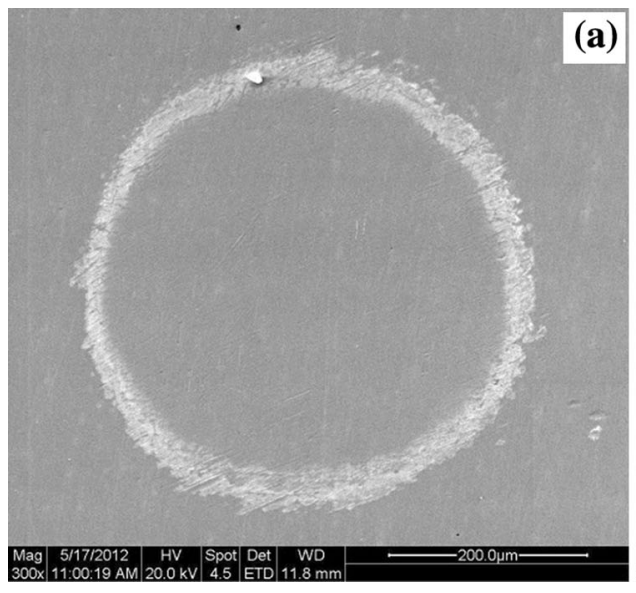

(b)
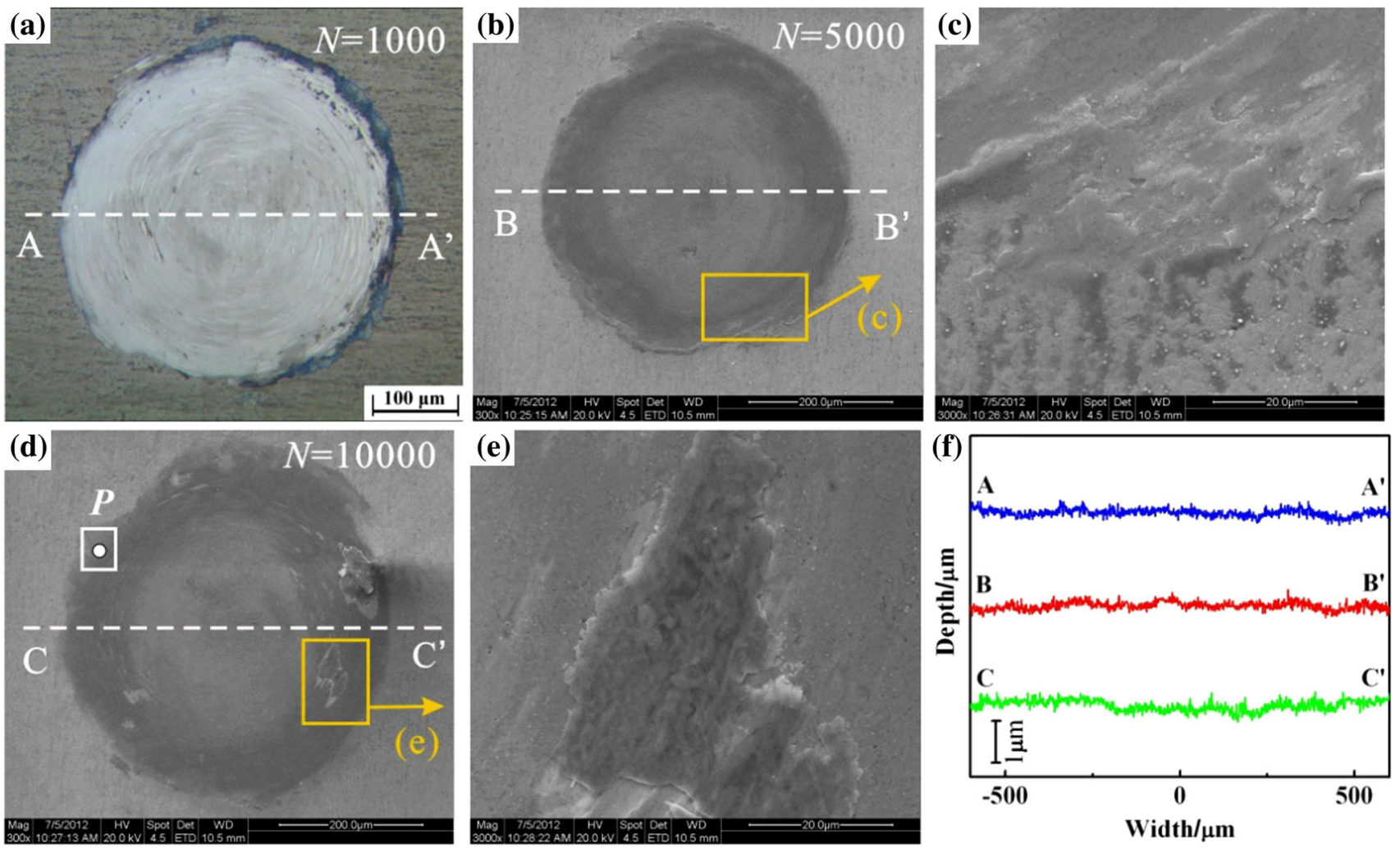

Fig. 8 Morphologies and 2D profile of the wear scars of DLC coatings under different cycles: $F_{n}=50 \mathrm{~N}, \theta=2.5^{\circ}$

no relative slip was found in the center contact interfaces and low relative slip at outer contact interfaces. This finding can be verified by $T-\theta$ curves in Fig. $2 \mathrm{a}$.

In SR, wear scars of DLC coatings were completely different from that in PSR. Damage occupied the whole contact zone. Figure 8 shows morphologies and 2D profile of wear scars of DLC coatings under different cycles $\left(\theta=2.5^{\circ}\right)$. After the number of cycles reached $N=1000$, damage was still slight and only a spot of debris was distributed at the edge of the wear scar (Fig. 8a). When the number of cycles increased to $N=5000$, because the slip gradually increased in radial direction, damage at the edge of contact zone was more severe than that in the center. In edge of the scar, ploughing appeared along the circumferential direction. At same time, a layer of debris can be seen outside of wear scar (Fig. 8b, c). When $n$ cycles increased to $10^{4}$, damage was more severe. DLC coatings were worn in the local area and damage occurred on interlay and nitride layers. DLC was delaminated directly from interlay, and debris was compacted to flats and covered the local area of the wear scar (Fig. 8d, e). Raman spectra of point $P$ showed that $\mathrm{G}$ peak shifted from 1574.9 
to $1583.5 \mathrm{~cm}^{-1}$ and $I_{\mathrm{D}} / I_{\mathrm{G}}$ ratio increased from 4.31 to 4.58 , compared with unwear surface (Fig. 12), which demonstrated an increase of $\mathrm{sp}^{2}$ content in the DLC coating. This result occurred due to shear stress and friction heat on the contact zone, which caused the progress of graphitization during friction process [31]. However, DLC coatings still provided continuous lubricating effect, which could be demonstrated by friction torque of DLC coatings. The coatings were still lower than that of LZ50 steel after 10,000 cycles. In sum, main mechanisms of DLC coatings

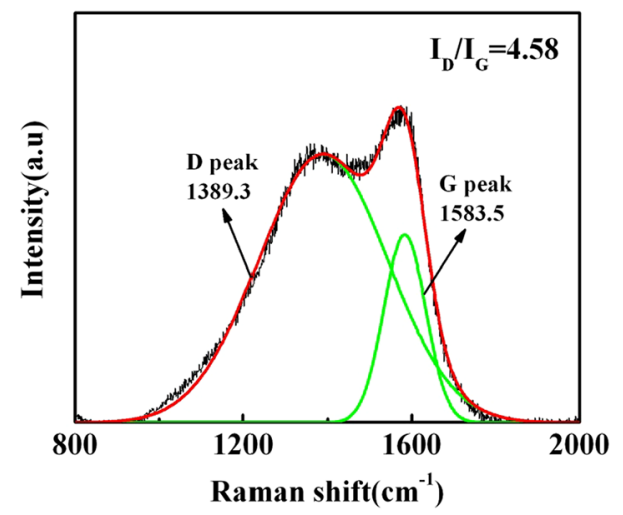

Fig. 9 Raman spectrum of the wear scars of DLC coatings: $F_{n}=50 \mathrm{~N}, \theta=2.5^{\circ}, N=10,000$ (Corresponding to $P$ point in Fig. 8d) were abrasive wear, delamination, and graphitization (Fig. 9).

When the angular displacement amplitude is $\theta=5^{\circ}$, both the LZ50 steel and DLC coatings ran in SR. To compare DLC coatings with the substrate (Fig. 10), the damage of the coating layer was obviously slighter than that of the substrate. The amount of debris could be found around the wear scar in LZ50, and only delamination occurred in the worn DLC. Furthermore, compared with the LZ50 steel in the wear area (Fig. 11) and the maximum wear depth (Fig. 12), the damage of the DLC coatings was

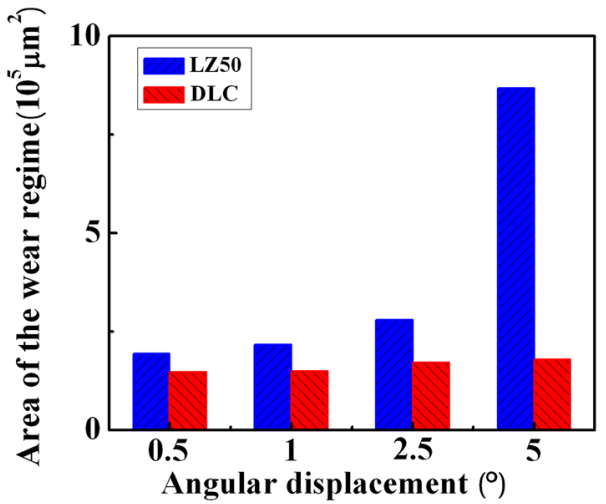

Fig. 11 Areas of the wear scars of DLC coatings and LZ50 steel substrate under different angular displacement amplitudes
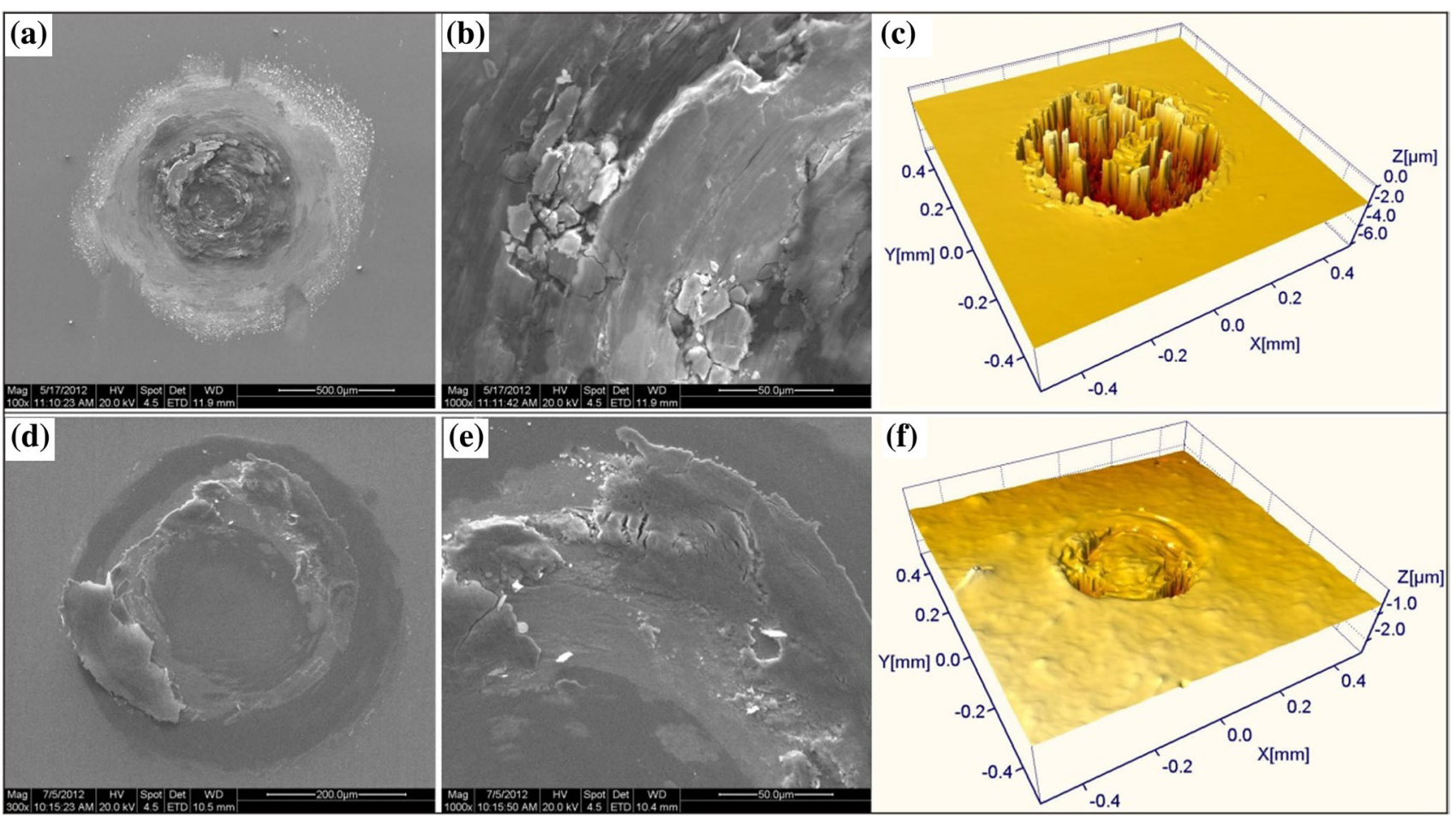

Fig. 10 SEM morphologies and 3D profile of wear scars of LZ50 (a-c) and DLC (d-f) coatings in SR: $\theta=5^{\circ}, N=10,000$ 
Fig. 12 Profiles of the wear scars of the DLC coatings and LZ50 steel substrate under different angular displacement amplitudes
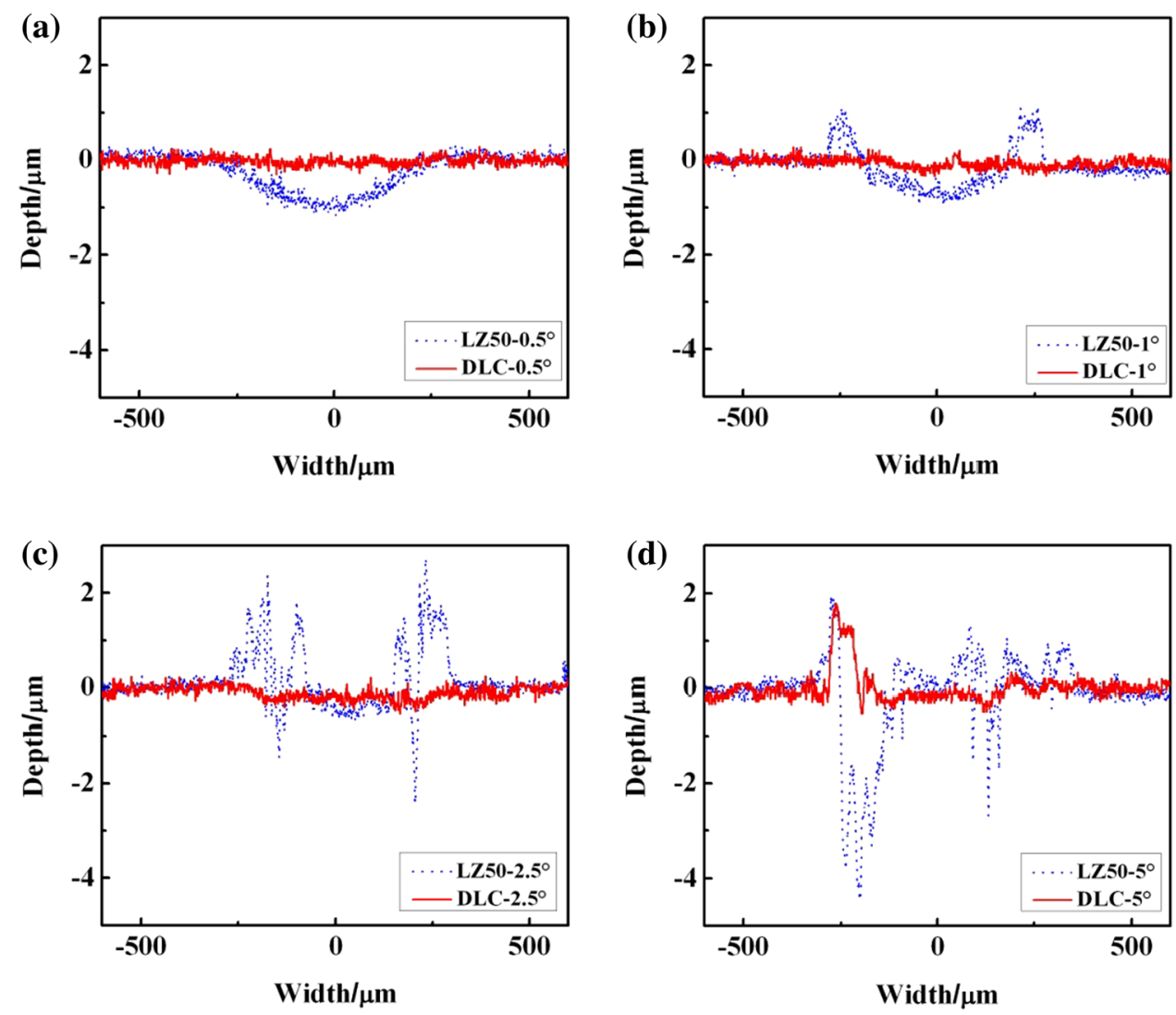

Fig. 13 Profiles of the worn GCr15 balls against DLC coatings and the LZ50 steel substrate under different angular displacement amplitudes

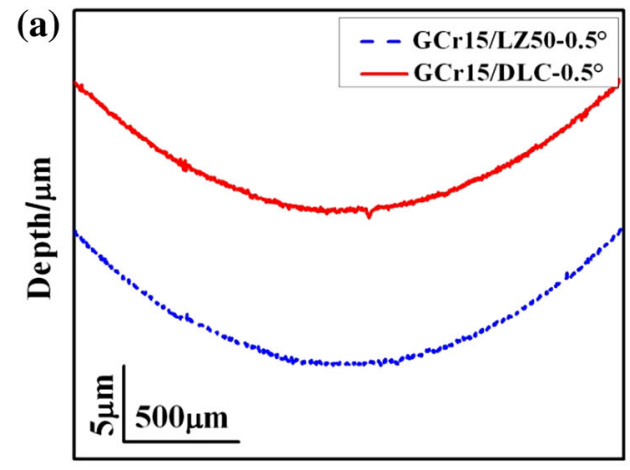

Width/ $\mu \mathrm{m}$

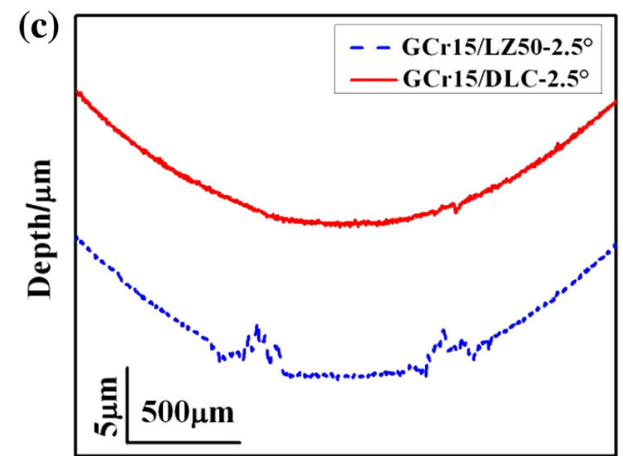

Width/ $\mu \mathrm{m}$

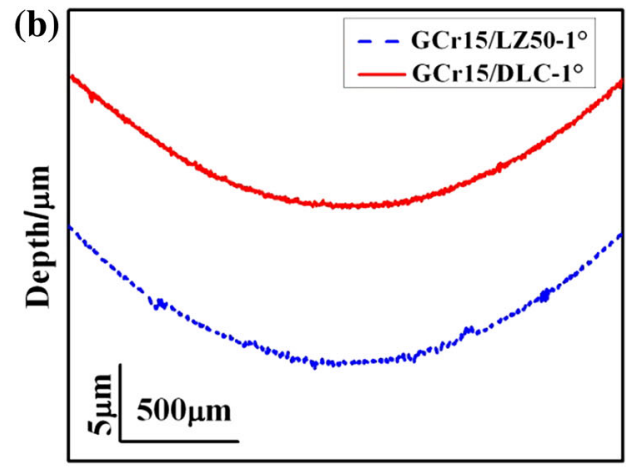

Width/ $\mu \mathrm{m}$

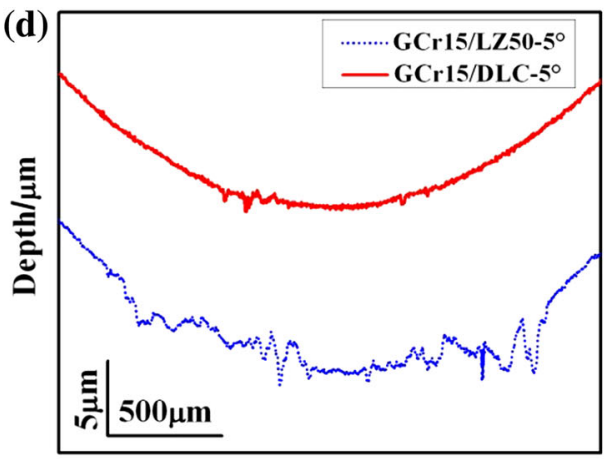

Width/ $\mu \mathrm{m}$ 
more notable than that of LZ50 steel under the same test conditions. Figure 13 shows the profiles of the wear scars of GCr15 balls for DLC coatings and the LZ50 steel substrate under different angular displacement amplitudes. At $\theta=0.5^{\circ}$ and $\theta=1^{\circ}$, damage was slight (Fig. 13a, b). Given that the hardness of the GCr15 ball was higher than that of LZ50, damage mainly occurred on the LZ50. However, both DLC coatings and the GCr15 ball had high hardness and the transfer layer easily formed on the contact interface, which could be the main reason for the slight damage. As mentioned above, the DLC coatings exhibited good capability for alleviating torsional fretting damage.

Torsional fretting or wear usually appear at joint whether human joints or ball joint in machine. The future work under medium action will be a good method. The medium and maybe show a very different result compare to dry condition in this paper-friction corrosion, effect of fluid to wear debris or tribochemistry. What performance can make nothing of the DLC in the lubricating medium, and it is worthy of our further and deep study.

\section{Conclusion}

DLC coatings with dense structures and high hardness and elastic modulus were prepared on LZ50 steel by duplex ion nitriding/IBAD treatment. The coating was tested by torsional fretting with a ball-plate contact. The following conclusions can be drawn from this study:

(1) For DLC coatings, only two torsional fretting running regimes (PSR and SR) were determined by the $T-\theta$ curves and the evolution of wear scar, i.e., MFR disappeared and SR shifted to lower angular displacement amplitudes.

(2) The friction torques of the DLC coatings were lower than that of LZ50 steel under the same fretting conditions because of the formation of a carbon transfer layer on the contact interface.

(3) DLC coatings exhibited good capability for alleviating torsional fretting damage. In PSR, the damage of the DLC coatings was slight and only exhibited a rolling mark. In SR, the damage mechanism of the DLC coatings combined with delamination, abrasive wear, and graphitization. The graphitization of DLC during the friction process can provide a continuous lubricating effect and reduce wear.

\section{References}

1. Waterhouse RB (1981) Fretting fatigue. Applied science, London

2. Zhou ZR, Vincent L (1997) Cracking induced by fretting of aluminum alloys. J Tribol ASME 119:36-42
3. Waterhouse RB (1972) Fretting corrosion. Pergamon, Oxford

4. Zhou ZR, Vincent L (1995) Mixed fretting regime. Wear 181-183:531-536

5. Zhu MH, Zhou ZR (2011) On the mechanisms of various fretting wear modes. Tribol Int 44:1378-1388

6. Zhu MH, Zhou ZR (2001) An experimental study on radial fretting behavior. Tribol Int 34:321-326

7. Cai ZB, Zhu MH, Zhou ZR (2010) An experimental study torsional fretting behaviors of LZ50 steel. Tribol Int 43:361-369

8. Cai ZB, Zhu MH, Zheng JF et al (2009) Torsional fretting behaviors of LZ50 steel in air and nitrogen. Tribol Int 42:1676-1683

9. Cai ZB, Zhang GA, Zhu YK et al (2013) Torsional fretting wear of a biomedical Ti6Al7 $\mathrm{Nb}$ alloy for nitrogen ion implantation in bovine serum. Tribol Int 59:312-320

10. Cai Z-B, Gao S-S, Zhu M-H et al (2011) Tribological behavior of polymethyl methacrylate against different counter-bodies induced by torsional fretting wear. Wear 270:230-240

11. Cai Z-B, Zhu M-H, Yang S et al (2011) In situ observations of the real-time wear of PMMA flat against steel ball under torsional fretting. Wear 271:2242-2251

12. Hicks J, Arnold A, Anderson F et al (2007) The effect of excessive tibial torsion on the capacity of muscles to extend the hip and knee during single-limb stance. Gait Posture 26:546-552

13. Hirakawa K, Toyama K, Kubotat M (1998) The analysis and prevention of failure in railway axles. Int J Fatigue 20:135-144

14. Waterhouse RB, Chivers TC (eds) (1994) Fretting fatigue. ESIS Publication, London

15. Zhou ZR, Vincent L (1999) Lubrication in fretting-a review. Wear 225-229:962-967

16. Zhu MH, Zhou ZR (2001) An investigation of molybdenum disulfide bonded solid lubricant coatings in fretting conditions. Surf Coat Int 141:240-245

17. Penkov Oleksiy V, Pukha Volodymyr E, Zubarev Evgeniy N et al (2013) Tribological properties of nanostructured DLC coatings deposited by C60 ion beam. Tribol Int 60:127-135

18. Suzuki M, Saito T, Tanaka A (2013) Tribological properties of DLC films against different steels. Wear 304:83-87

19. Zhiqiang Fu, Jian Sun, Chengbiao Wang et al (2013) Tribological performance of DLC coatings deposited by ion beam deposition under dry friction and oil lubricated conditions. Vacuum 94:14-18

20. Tillmann W, Vogli E, Hoffmann F (2009) Wear-resistant and low-friction diamond-like-carbon (DLC)-layers for industrial tribological applications under humid conditions. Surf Coat Int 204:1040-1045

21. Li CX, Sun Y, Bell T (2000) Factors influencing fretting fatigue properties of plasma-nitrided low alloy steel. Mater Sci Eng A 292:18-25

22. She DS, Yue W, Fu ZQ et al (2013) The effect of nitriding temperature on hardness and microstructure of die steel pretreated by ultrasonic cold forging technology. Mater Des 49:392-399

23. Yue W, Fu Z, Wang S et al (2014) Tribological synergistic effects between plasma nitrided 52100 steel and molybdenum dithiocarbamates additive in boundary lubrication regime. Tribol Int 74:72-78

24. Liu C, Yue W, Wang C et al (2012) The interactions between sulfur-nitrided layer on steel surface and MoDTC lubricating additive and their effects on tribological performance. Tribol Lett 47:313-322

25. Robertson J (2012) Diamond-like amorphous carbon. Mater Sci Eng R 37:129-281

26. Yue Wen, Gao Xiaocheng, Liu Yuandong et al (2011) Tribological properties of sulfurized-nitrided layer prepared by a twostep method. Vacuum 85:1011-1016 
27. Savvides N, Bell TJ (1992) Microhardness and Young's modulus of diamond and diamond-like carbon films. J Appl Phys 72:2791-2796

28. Fouvry S, Kapsa P, Zahouani H et al (1997) Wear analysis in fretting of hard coatings through a dissipated energy concept. Wear 203-204:393-403

29. Fouvry S, Paulin C (2014) An effective friction energy density approach to predict solid lubricant friction endurance: application to fretting wear Wear 319:211-216
30. Meiboom S, Hewitt RC (1977) Rotational viscosity in the smectic phases of terephthal-bis-butylaniline (TBBA). Phys Rev A 15:2444-2453

31. Voevodin AA, Muratore C, Aouadic SM (2014) Hard coatings with high temperature adaptive lubrication and contact thermal management: review. Surf Coat Int 257:247-265 